\title{
Relationship between Cardiac Cycle Length and Ventricular Relaxation Rate in the Chick Embryo
}

\author{
VASANA CHEANVECHAI, SHARON F. HUGHES, AND D. WOODROW BENSON, JR \\ Division of Cardiology, Children's Memorial Hospital, Northwestern University Medical School and the Feinberg \\ Cardiovascular Research Institute, Chicago, Illinois 60614
}

\begin{abstract}
We hypothesized that during chick embryo cardiac development, cycle length decrease (heart rate increase) may be associated with developmental changes in the ventricular relaxation rate constant, $\tau$, which can be estimated from ventricular pressure decrease by the function $P(t)=P_{0} e^{-t / 7}$, where $P_{0}=$ pressure at the time of minimum time derivative of ventricular pressure and $t=$ time. Natural logarithm conversion results in a linear relation between $\ln P(t)$ and $t$ with slope of $-1 / \tau$. We determined $\tau$ in Hamburger-Hamilton stage 17-27 (d 3-5) embryos $(n=35)$ at intrinsic cycle length and in stage 24 embryos $(n=5)$ during cardiac cycle length perturbations with the hot-cold probe technique. Regression analysis of In $P(t)$ from the minimum $d P / d t$ until $t=30 \mathrm{~ms}$ was used to estimate $\tau$. Intrinsic cycle length decreased during development from stage 17 to 27 and $\tau$ decreased linearly as cycle length decreased $(r=0.39, p<0.005)$. Thus, during early development, both $\tau$ and cycle length decrease (relaxation rate and heart rate increase). In stage 24 embryos, cycle length perturbation yielded a nonlinear cycle lengthdependent relation with $\tau$, with a plateau at cycle lengths less than intrinsic, i.e. $\tau$ decreased with cycle length decrease to intrinsic cycle length, then $\tau$ plateaued with further cycle length decrease. These findings demonstrate that ventricular relaxation rate is both maturation and cycle length dependent. The plateau effect of the $\tau$-cycle length relationship may be due to developmental limitations of the calcium transport system. (Pediatr Res 31: 480-482, 1992)
\end{abstract}

\section{Abbreviations}

$\mathrm{dP} / \mathrm{dt}$, time derivative of ventricular pressure

In the chick embryo, cardiac cycle length decreases (i.e. heart rate increases) during development and the associated changes in systolic cardiac performance (e.g. aortic blood flow, stroke volume, and cardiac work) have been well described (1). However, the relationship between diastolic function and developmental heart rate changes has not been completely evaluated in the chick embryo.

Ventricular relaxation rate is an important attribute of diastolic ventricular function, and we wondered if there might be developmental changes in ventricular relaxation as characterized by $\tau$, the time constant for isovolumic pressure decline (2). We hypothesized that the developmental changes in the relaxation rate are directly related to changes in cardiac cycle length. To

Received August 19, 1991 ; accepted December 16, 1991

Correspondence: D. Woodrow Benson, Jr., M.D., Ph.D., Division of Cardiology, 2300 Children's Plaza, Chicago, IL 60614.

Supported in part by a grant from the American Heart Association (89-0886) and the Walden W. and Jean Young Shaw Foundation. test this hypothesis, we evaluated the relationship between cycle length and ventricular relaxation rate in chick embryos during early cardiac development. We found $\tau$ to be dependent on both developmental stage and cardiac cycle length.

\section{MATERIALS AND METHODS}

Fertile White Leghorn chicken eggs were incubated blunt end up at $38^{\circ} \mathrm{C}$ in a forced-draft incubator to Hamburger-Hamilton stages 17 (d 3) to 28 (d 5) (3). An egg was removed from the incubator and placed in a heated sand bath (Bioengineering Department, Medical College of Georgia) under a radiant warmer to maintain a constant egg temperature of $37-38^{\circ} \mathrm{C}$ throughout the experiment. The egg was observed with a dissecting microscope; a window in the shell was created, and the extraembryonic and pericardial membranes were removed to expose the embryo.

Ventricular pressure measurement. Ventricular pressure was measured with a servo-null micropressure system (model 900; WP Instruments, New Haven, CT) from a drawn glass micropipette (tip diameter $=7 \mu \mathrm{m}$ ) inserted directly into the ventricle. The micropressure system had been validated against a water column, and in vitro measurements were found to be linear over a range of 0 to $6.67 \mathrm{kPa}(0-50 \mathrm{~mm} \mathrm{Hg})$. Signals were amplified using a direct current amplifier (model 319D; BMT Instruments, Southhold, NY) and digitally recorded at 500 samples/s. Zero transtip pressure was established by immersing the electrode in the extraembryonic fluid at the level of the ventricle. The $\mathrm{dP} / \mathrm{dt}$ was derived from the pressure curve using numerical differentiation (Computerscope; RC Electronics, Santa Barbara, CA) (Fig. 1).

Ventricular pressure measurements were obtained at intrinsic cardiac cycle length in stage 17-27 chick embryos $(n=35)$ and during cycle length perturbation in stage 24 embryos $(n=5)$. Cardiac cycle length was altered using a $1-\mathrm{mm}$ sterile stainless steel probe previously cooled (to increase cycle length) or heated (to decrease cycle length) in a water bath (4). The probe was then placed on the sinus venosus for $10 \mathrm{~s}$ to shorten or prolong the cycle length. Use of the thermal probe directly on the sinus venosus limits the temperature effects to the heart without affecting the systemic vascular resistance, presumably resulting in an isolated cycle length change.

Any deformed embryos or embryos that bled during the procedure were discarded. Records from embryos in which the baseline pressure measurement was not stable throughout the experiment or embryos that did not recover within $10 \%$ of the baseline cycle length after perturbation were also discarded.

Relaxation rate constant. The ventricular relaxation rate constant, $\tau$, was quantified by fitting the time course of pressure decline with an exponential function of time

$$
\mathrm{P}(\mathrm{t})=\mathrm{P}_{0} \mathrm{e}^{-\mathrm{t} / \tau}
$$

where $P(t)$ is ventricular pressure at time $t, P_{0}$ is the ventricular pressure at the end of ventricular ejection, $t$ is the time, and $\tau$ is 

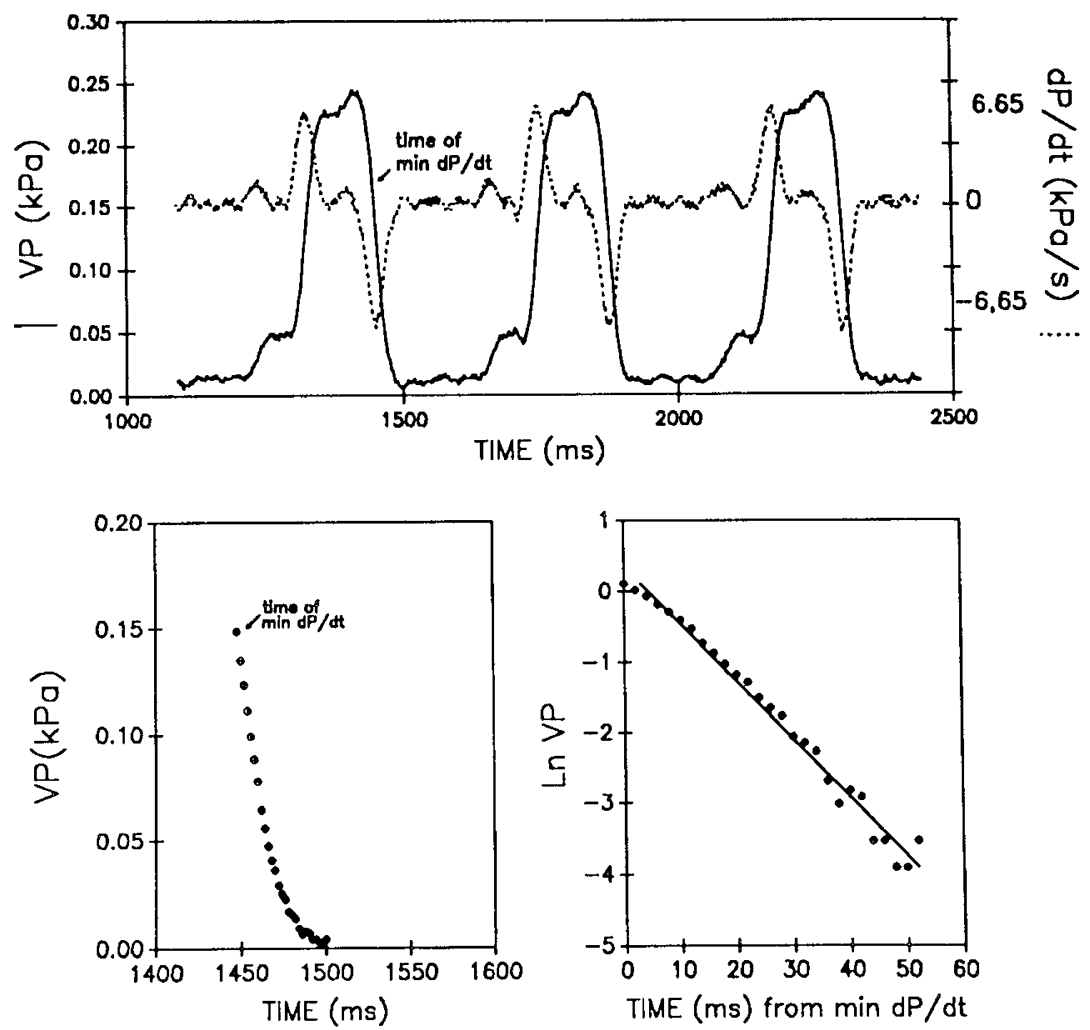

Fig. 1. Top panel, The time course of ventricular pressure fall superimposed on the time course of its derivative is shown. Lower left panel, The time course of ventricular pressure from time of minimum $\mathrm{dP} / \mathrm{dt}$ until $50 \mathrm{~ms}$ later is depicted. Lower right panel, Natural logarithm conversion of ventricular pressure is depicted. The slope of the line is inversely related to $\tau$ (slope $=-1 / \tau$ ).

the time constant of ventricular relaxation. Taking the natural logarithm of both sides of the above equation yields a linear relation

$$
\ln \mathrm{P}(\mathrm{t})=-\mathrm{t} / \tau+\ln \mathrm{P}_{0}
$$

with a slope of $-1 / \tau$. An increase in $\tau$ demonstrated by a decrease in slope indicates a reduction in the relaxation rate. A decrease in $\tau$ marked by an increase in slope, on the other hand, reflects a faster relaxation rate.

Previous studies have noted that the above equation implicitly assumes that at full relaxation of the ventricle without filling the pressure would fall asymptotically to zero (2). It has been shown, however, that there is no significant difference between the time constant calculated with an assumed asymptote of zero and the time constant using the measured pressure asymptote (5).

Data analysis. Isovolumic relaxation begins with the end of ventricular ejection and ends with the onset of ventricular filling. Minimum $\mathrm{dP} / \mathrm{dt}$ has been shown to reliably predict the end of ventricular ejection (6), but we could not determine the onset of ventricular filling, which we estimated to be $30-50 \mathrm{~ms}$. Therefore, linear regression analysis of the pressure logarithm versus time was performed in triplicate from minimum $\mathrm{dP} / \mathrm{dt}$ until $\mathrm{t}=30$, 40 , and $50 \mathrm{~ms}$. In this study, $\tau$ values calculated using 40 or 50 $\mathrm{ms}$ did not vary considerably from the $30 \mathrm{~ms}$ values. In addition, pressures measured at 40 and $50 \mathrm{~ms}$ often yielded values falling below the baseline pressure, which resulted in undefinable natural logarithmic values. Thus, $\tau$ values reported here were calculated using time from minimum $\mathrm{dP} / \mathrm{dt}$ to time $=30 \mathrm{~ms}$.

Values for $\tau$ were determined for five beats per embryo at intrinsic heart rate for the stage 17-28 embryos. For each of the stage 24 embryos, $\tau$ was determined for five beats at intrinsic, increased, or decreased heart rates.

\section{RESULTS}

For the 35 embryos, intrinsic cycle length (heart rate) ranged from $344 \mathrm{~ms}(174 \mathrm{bpm})$ to $660 \mathrm{~ms}(80 \mathrm{bpm})$. Peak systolic pressure ranged from 0.13 to $0.67 \mathrm{kPa}$. Heart rate perturbation performed in five stage 24 embryos yielded a minimum and maximum cycle length of $294 \mathrm{~ms}$ (204 bpm) and $1028 \mathrm{~ms}$ (58 $\mathrm{bpm}$ ) during hot and cold probe application, respectively.

Both intrinsic cycle length and $\tau$ decreased during development from stage 17 to 28 (Fig. 2). A developmentally younger embryo has a slower intrinsic heart rate (longer cycle length) as well as a slower relaxation rate as indicated by a larger $\tau$ value. Thus, in chick embryo, $\tau$ decreases as developmental cycle length decreases (Fig. 3).

To further evaluate this apparent cycle length-dependent relationship of $\tau$, we looked at $\tau$ changes within one single stage of development. Changes in $\tau$ observed in the stage 24 chick embryos with cycle length perturbations demonstrated the effect of changes in cycle length without accompanying morphologic changes of development. In stage 24 embryos, cycle length perturbation yielded a nonlinear $\tau$-cycle length-dependent relationship with a plateau at cycle lengths less than or equal to intrinsic cycle lengths. Results from a single embryo are shown in Figure 4.

\section{DISCUSSION}

The principal finding in this study is that in chick embryos ventricular relaxation rate is both maturation and cycle length dependent. In the mature heart, ventricular relaxation rate is an important determinant of the early diastolic ventricular pressurevolume relation (2), and it has been used as a measure of myocardial relaxation (7). Because the rate of myocardial relaxation is determined by the rate of removal of calcium from myofibrillar proteins, the developmental decrease in $\tau$ suggests maturation in the calcium transport system. The plateau effect of the $\tau$-cycle length relationship in the stage 24 embryos may be due to developmental limitations of the calcium transport system.

In the stage 24 chick embryo, the cardiovascular system is 

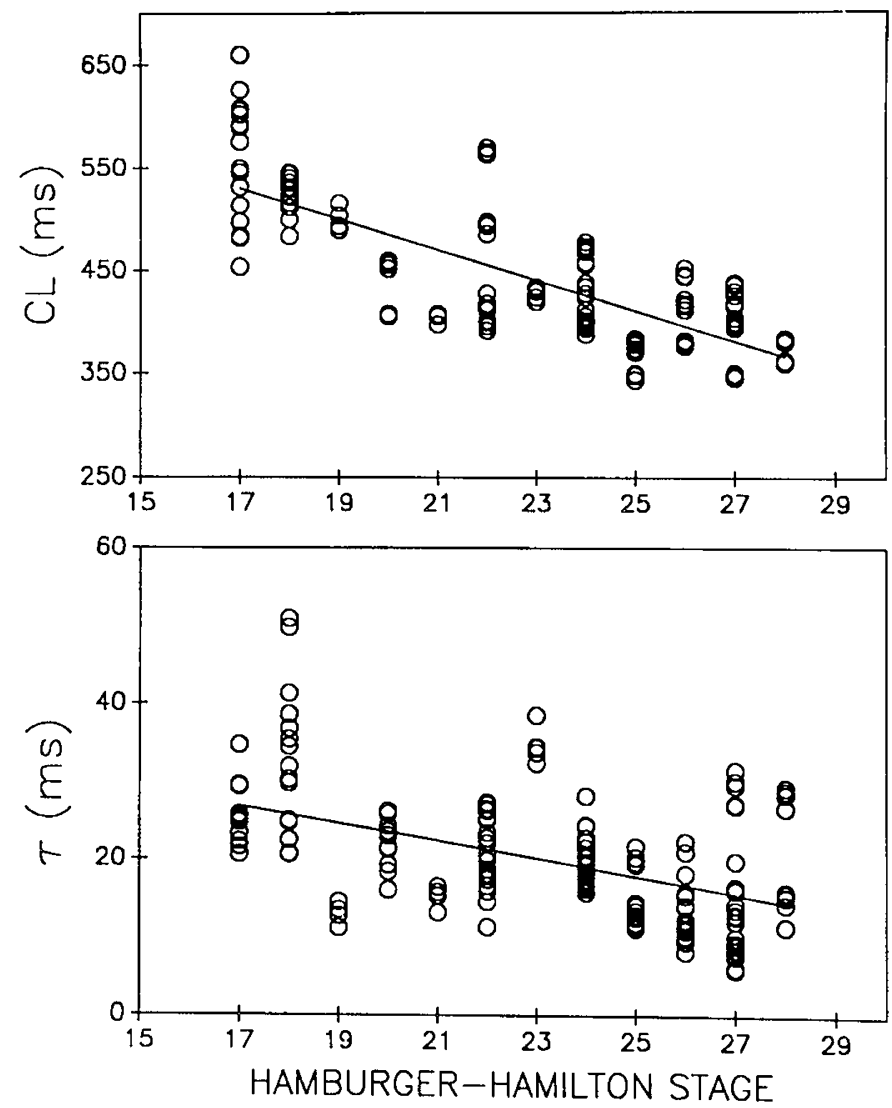

Fig. 2. Relation of cycle length $(C L)$ (top panel) and relaxation rate $(\tau)$ (bottom panel) to Hamburger-Hamilton stage. Both $\tau(r=0.49$, slope $=-1.41, p<0.05)$ and cycle length $(r=-0.77$, slope $=-15.0, p<0.05)$ decrease during development.

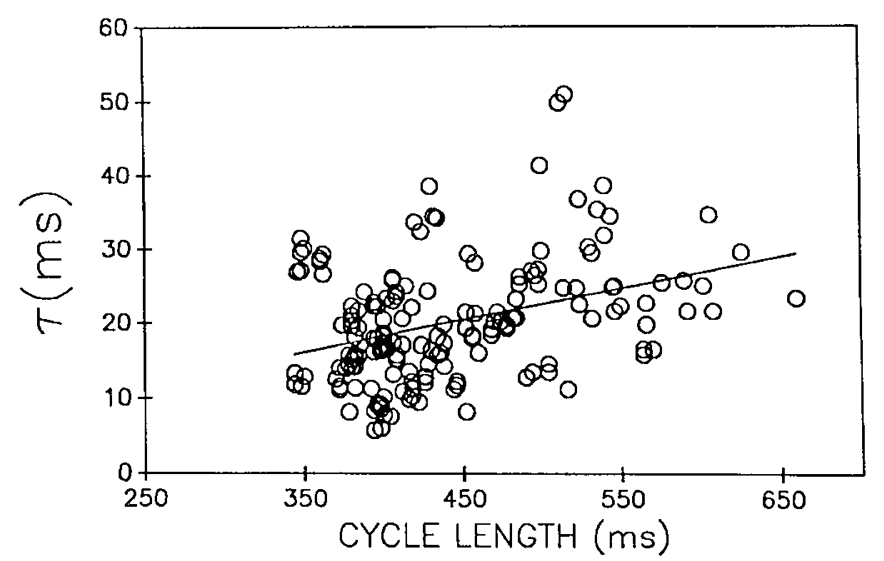

Fig. 3. Relationship of $r$ to developmental changes in cycle length. As shown, $\tau$ changes with developmental cycle length in stage 17-28 chick embryos $(r=0.39$, slope $=0.047, p<0.05)$.

"tuned" so that maximal aortic flow occurs at the intrinsic heart rate (4). The extent to which the heart rate-relaxation rate relationship affects ventricular filling has not been completely determined in the developing heart. In a recent study of diastolic ventricular filling characteristics, the proportion of passive filling (that which occurs before atrial contraction) and active filling (in association with atrial contraction) changed as the ventricle developed from a smooth-walled tube to a sponge-like chamber (7). When comparable heart rates were achieved by hypothermia or hyperthermia, passive filling dominated in young embryos, whereas active filling dominated later in development as ventricular trabeculae developed. These changes in filling characteristics and the developmental increase in ventricular end-diastolic pres-

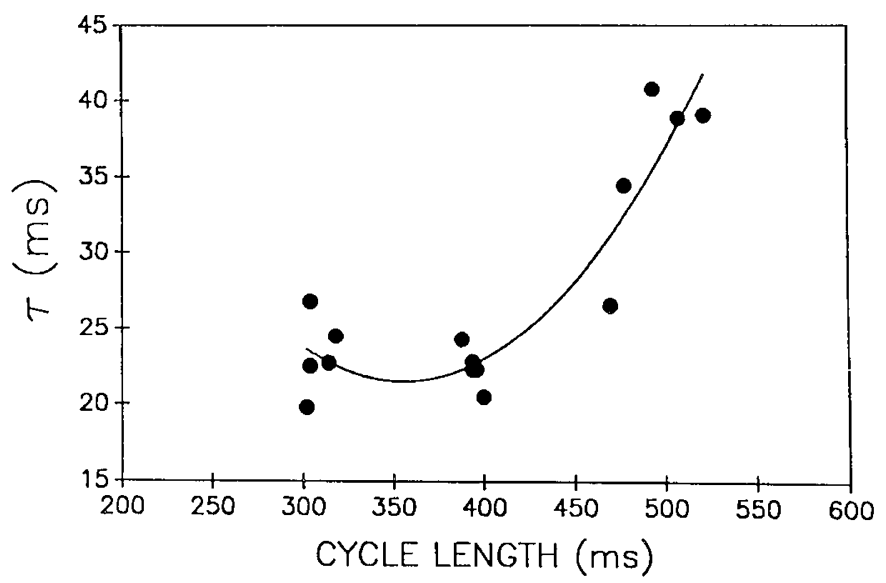

Fig. 4. Relationship of $\tau$ to perturbed heart rate in a stage 24 embryo. A quadratic relationship $\left(R^{2}=0.87\right)$ has been modeled. When cycle length is prolonged, $r$ is increased, but when cycle length is shortened to less than intrinsic, the $\tau$-cycle length relation appears to plateau.

sure have been interpreted as showing a developmental decrease in ventricular compliance (8). It is possible that temperatureinduced changes in afterload $(9,10)$ may affect the rate and extent of ventricular relaxation (2) and, thereby, affect the ventricular filling characteristics. Alternatively, alterations in myocardial temperature may directly affect the calcium transport system (11) and, thereby, affect ventricular filling characteristics.

In the mature heart, increases in the rate of removal of calcium from the myofibrils are mediated partly by increased calcium transport by the sarcoplasmic reticulum, an important determinant of relaxation (7). In the chick embryo, however, the sarcoplasmic reticulum plays a limited role in the calcium transport until during or after hatching, and sodium-calcium exchange appears to play a greater role in calcium transport early in development (12). Differences in the ability of these calcium transport systems to sequester calcium may contribute to the differences in the functional responses of the heart during development and cycle length perturbations. The basis for these developmental differences must be investigated further and may prove to be associated with the age-related changes in the function and capacity of the calcium transport system. The ability of the mature myocardium to alter relaxation time may be greater than that of the immature heart due to the different transport mechanisms that may be active as maturation advances.

\section{REFERENCES}

1. Hu N, Clark EB 1989 Hemodynamics of the stage 12 to stage 29 chick embryo. Circ Res 65:1665-1670

2. Gilbert JC, Glantz BA 1989 Determinants of left ventricular filling and of the diastolic pressure-volume relation. Circ Res 64:827-852

3. Hamburger V, Hamilton HL 1951 A series of normal stages in the development of the chick embryo. J Morphol 88:49-92

4. Cuneo B, Hughes S, Benson Jr DW 1991 Heart rate perturbation in the chick embryo: a comparison of two methods. Am J Physiol 260:H1864-H1869

5. Yellin EL, Hori M, Yoran C, Sonnenblick EH, Gabbay S, Frater RWM 1986 Left ventricular relaxation in the filling and nonfilling intact canine heart. Am J Physiol 250:H620-H629

6. Abel FL 1981 Maximal negative dP/dt as an indicator of end of systole. Am J Physiol 240:H676-H679

7. Kaufman TM, Horton JW, White DJ, Mahony L 1990 Age-related changes in myocardial relaxation and sarcoplasmic reticulum function. Am J Physiol 259:H309-H316

8. Hu N, Connuck DM, Keller BB, Clark EP 1991 Diastolic filling characteristics in the stage 12-27 chick embryo ventricle. Pediatr Res 29:334-337

9. Nakazawa M, Clark EB, Hu N, Wispe J 1985 Effect of environmental hypothermia on vitelline artery blood pressure and vascular resistance in the stage 18,21 , and 24 chick embryo. Pediatr Res 19:651-654

10. Nakazawa M, Miyagawa S, Takao A, Clark EB, Hu N 1986 Hemodynamic effects of environmental hyperthermia in stage 18,21, and 24 chick embryos. Pediatr Res 20:1213-1215

11. Martonsi A, Feretos R 1964 Sarcoplasmic reticulum. J Biol Chem 239: 648-658

12. Vetter R, Will H 1986 Sarcolemmal Na-Ca exchange and sarcoplasmic reticulum uptake in developing chick heart. J Mol Cell Cardiol 18:1267-1275 